\title{
Probing the Effects of Electron Channelling on EDX Quantification
}

\author{
Katherine E. MacArthur ${ }^{1}$, Hamish G. Brown ${ }^{2}$, Scott D. Findlay ${ }^{2}$, Leslie J. Allen ${ }^{3}$
}

1. The Ernst Ruska Centre for Microscopy and Spectroscopy with Electrons, Forschungszentrum Jülich GmbH, Jülich, Germany.

2. School of Physics and Astronomy, Monash University, Melbourne, Australia.

${ }^{3 .}$ School of Physics, University of Melbourne, Melbourne, Australia.

A myriad of advances in microscope stability, aberration correction and improved detectors now make it routinely possible to achieve atomic resolution energy dispersive X-ray (EDX) mapping [1], showing atomic-scale qualitative detail as to where the elements reside within a sample. Whenever we analyse crystals down a low-order zone axis we are exploiting electron channelling to provide us with atomic resolution. The aligned columns act like miniature lenses, providing a focusing effect on the electron beam. This is beneficial because it causes our convergent beam to stay more focused onto one atomic column, providing increased signal from an aligned column of atoms than we would expect just by summing the intensity of each of the component atoms [2]. However, the focusing effect further means the contribution from atoms at different depths differs, a phenomenon often referred to as the 'TopBottom Effect', complicating the quantitative interpretation.

Figure 1 plots the X-ray signal from a single column in a single element crystal, as a function of both the column thickness and of sample tilt away from the on-axis condition, normalized by the X-ray signal from a single atom. Therefore, a value of 1 indicates a linear signal and an increase of the fractional Xray intensities with increasing crystal thickness show that the 'lighting-up', i.e. the enhancement of the signal resulting from the focusing/channeling effect observed in HAADF imaging, also occurs in the EDX case. Because this effect is dependent on atom type, it will also have an effect on quantification. Moreover, figure 1 shows that the enhancement effect reduces with increasing tilt: by a tilt of $2^{\circ}$, the signal from each atom along the column is much closer to 1 , suggesting small tilt as a strategy for robust counting of atoms in a column in elemental crystals. Similar results have been shown by Lugg et al. for unit cell averages in $\mathrm{SrTiO}_{3}$ [3].

Determining composition in mixed-species columns presents a similar challenge. Focusing specifically on the simplest composition case of a binary system we investigate the effect of ordering on the X-ray signal. Taking the scenario of a bimetallic column containing 5 Pt atoms and $2 \mathrm{Ni}$ atoms ( 7 atoms in total) there are 21 different possible configurations. This range of configurations produces a range different X-ray signals which most likely translates to an uncertainty in determination of sample composition, if unknown. Figure 2, shows how the X-ray signal from the different configurations changes with sample tilt away from a $<110>$ zone axis. The further one tilts away from a low-order zone-axis the closer the signal gets to the non-channelling case and the spread in values between different configurations decreases. Therefore, the more we intentionally tilt the sample the more accurate our composition determination will be, at least for small sample tilts. At larger tilts, the tilted columns may overlap in projection and the signals of individual columns can no longer be separated. Between these two competing effects lies a minimum for the most accurately achievable composition quantification. With accurate knowledge of the sample composition, we can begin to envisage deconvolving the configuration information contained in the on-axis data $[4,5]$. 


\section{References:}

[1] AJ D'Alfonso et al, Phys. Rev. B 81 (2010) p.100101

[2] H E et al, Ultramicroscopy 133 (2013) p. 109-119

[3] NR Lugg et al, Ultramicroscopy 151 (2015) p. 150-159

[4] B Esser et al, Phys. Rev Lett. 117 (2016) p. 176101

[5] This research was supported under funding from the Helmholtz Association in Germany and the Australian Research Council's Discovery Projects funding scheme (Project DP140102538).
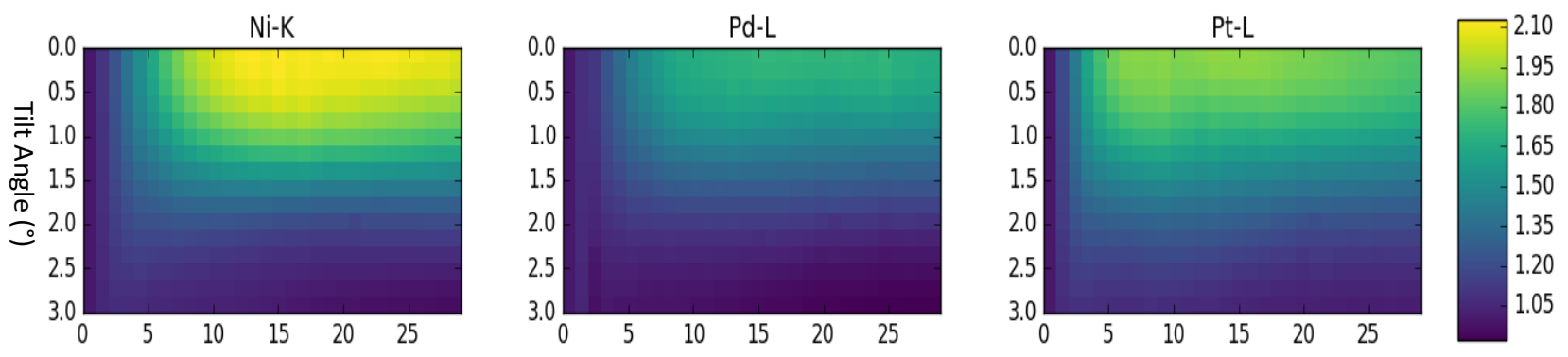

Column Thickness in Atoms

Figure 1. Simulated curves showing the fractional increase of X-ray counts resulting from electron channelling from each atomic column a single element FCC crystal, as a function of both the column thickness and of sample tilt away from the on-axis condition, for the Ni-K, Pd-L and Pt-L X-ray lines, respectively. The relative change in X-ray intensity varies with the element type as different weight atoms provide a different lensing effect on the electrons.
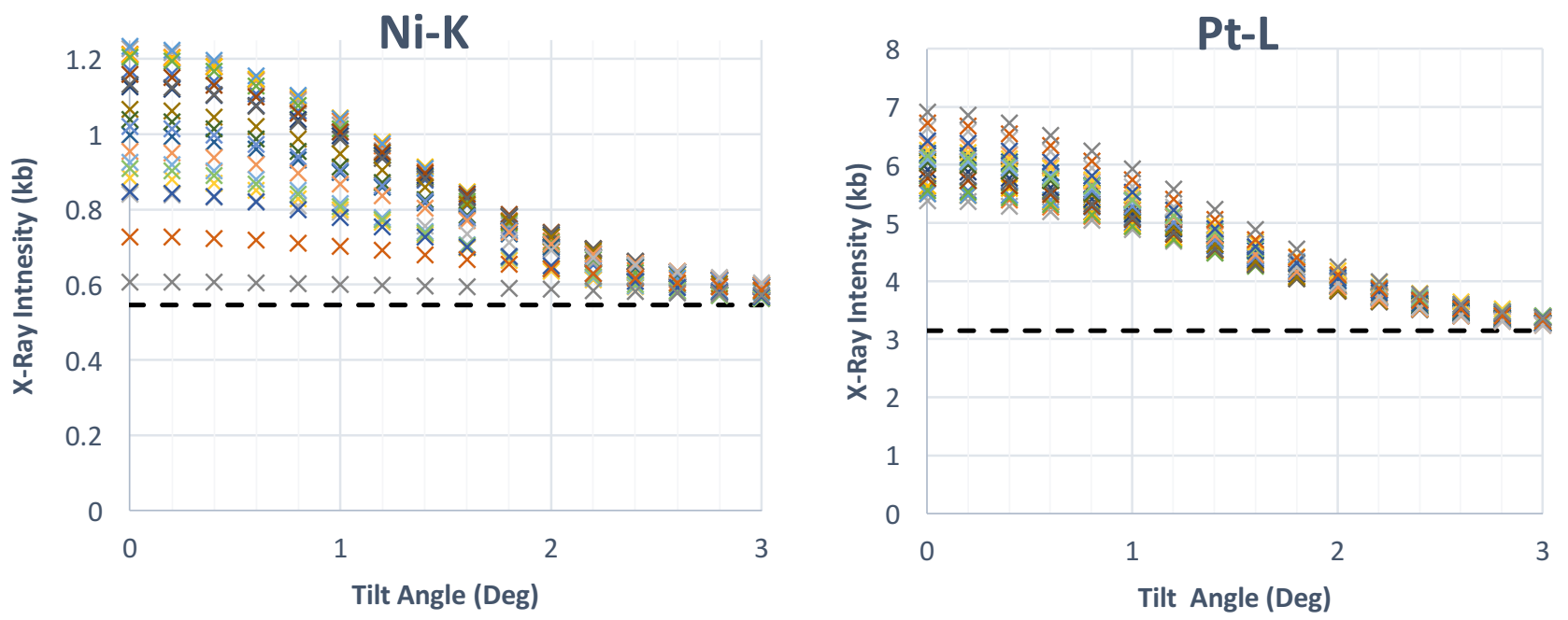

Figure 2. Plots showing the predicted X-ray intensity for a 7-atom thick column containing 5 atoms of $\mathrm{Pt}$ and 2 atoms of $\mathrm{Ni}$ for various configurations (denoted by different data point colour). The simulations are periodic in 2-dimensions to forming a layered crystal. The simulated intensity varies greatly depending both on sample tilt and on the arrangement of the atoms within the column. At higher tilt angles the integrated intensity (or cross section) of a column approaches the non-channelling case (black dashed line) regardless of the atomic configuration. The Ni-K signal appears to be much more affected by the presence of heavy $\mathrm{Pt}$ atoms than vice versa. 